\title{
Understanding packet loss for sound monitoring in a smart stadium loT testbed
}

\author{
Suzanne Little, Dian Zhang, \\ Camille Ballas, Noel E. O’Connor \\ Insight Centre for Data Analytics \\ Dublin City University \\ Dublin, Ireland \\ suzanne.little@dcu.ie \\ Niall Moran, \\ Mike Myers, Clare Dillon, \\ Microsoft Ireland \\ niall.moran@microsoft
}

\author{
David Prendergast, \\ Keith Nolan, Brian Quinn, \\ Intel Ireland Ltd. \\ david.k.prendergast@intel.com
}

Tomás Meehan

Gaelic Athletic Association

Dublin, Ireland

\begin{abstract}
The Smart Stadium for Smarter Living project provides an end-toend testbed for IoT innovation through a collaboration between Croke Park Stadium in Dublin, Ireland and Dublin City University, Intel and Microsoft. This enables practical evaluations of IoT solutions in a controlled environment that is small enough to conduct trials but large enough to prove and challenge the technologies. An evaluation of sound monitoring capabilities during the 2016 sporting finals was used to test the capture, transfer, storage and analysis of decibel level sound monitoring. The purpose of the evaluation was to use existing sound level microphones to measure crowd response to pre-determined events for display on big screens at half-time and to test the end-to-end performance of the testbed. While this is not the specific original purpose of the sound level microphones, it provided a useful test case and produced engaging content for the project. Analysis of the data streams showed significant packet loss during the events and further investigations were conducted to understand where and how this loss occurred. This paper describes the smart stadium testbed configuration using Intel gateways linking with the Azure cloud platform and analyses the performance of the system during the sound monitoring evaluation.
\end{abstract}

\section{CCS CONCEPTS}

- Information systems $\rightarrow$ Data analytics; Sensor networks; Data stream mining; • Networks $\rightarrow$ Network performance analysis; Middle boxes / network appliances;

\section{KEYWORDS}

Internet-of-Things, testbed, Azure platform, sound monitoring

ACM Reference format:

Suzanne Little, Dian Zhang, Camille Ballas, Noel E. O'Connor, David Prendergast, Keith Nolan, Brian Quinn,, Niall Moran, Mike Myers, Clare Dillon,

Permission to make digital or hard copies of part or all of this work for personal or classroom use is granted without fee provided that copies are not made or distributed for profit or commercial advantage and that copies bear this notice and the full citation on the first page. Copyrights for third-party components of this work must be honored.

For all other uses, contact the owner/author(s).

FAILSAFE2017, Nov 2017, Delft, The Netherlands

(c) 2017 Copyright held by the owner/author(s).

ACM ISBN 123-4567-24-567/08/06

https://doi.org/10.475/123_4 and Tomás Meehan. 2017. Understanding packet loss for sound monitoring in a smart stadium IoT testbed. In Proceedings of ACM SenSys, Delft, The Netherlands, Nov 2017 (FAILSAFE2017), 6 pages.

https://doi.org/10.475/123_4

\section{SMART STADIUM FOR SMARTER LIVING TESTBED}

A major challenge in advancing work in Internet-of-Things (IoT) and smart cities research is the difficulty in moving projects from the lab to real environments [4]. Tests conducted in small, artificial scenarios using data samples are valuable for developing solutions but rarely provide sufficient data to give confidence for deployment at city scale. The Smart Stadium project is a collaboration between the GAA (Ireland's national sporting body), Dublin City University (DCU), Intel, and Microsoft to further innovation around IoT ${ }^{1}$. The Smart Stadium testbed setup at Croke Park Stadium in Dublin provides a scalable option that is small enough to manageably trial but large enough to prove the effectiveness of new technologies. Intel has strategically positioned sensors and gateways throughout the stadium to enable a range of environmental monitoring, safety, and fan experience use cases. These edge gateways compute and communicate with the sensors, collecting diverse types of data and storing and processing them on the Microsoft Azure cloud platform.

Figure 1 shows a high-level overview of the testbed architecture. The testbed currently consists of sensors including a simple camera, environmental sensors and sound monitoring microphones together with five Intel gateways linked to Microsoft's Azure cloud platform for integration and analytics. The Smart Stadium project focusses on practical proof of concept activities that are relevant for Croke Park including modelling crowd movement, monitoring sunlight exposure on the pitch, measuring wind speed and rainfall and measuring the noise made by the crowd.

This paper describes the experience of creating a testbed system for Internet-of-Things and Smart Cities technologies in Croke Park Stadium and describes the learnings from an end-to-end test conducted around sound level analysis during major match days focussing on the packet loss issues. As expected we encountered physical, network and data issues that cause significant packet loss

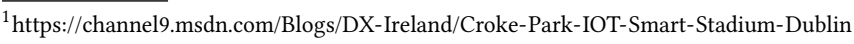




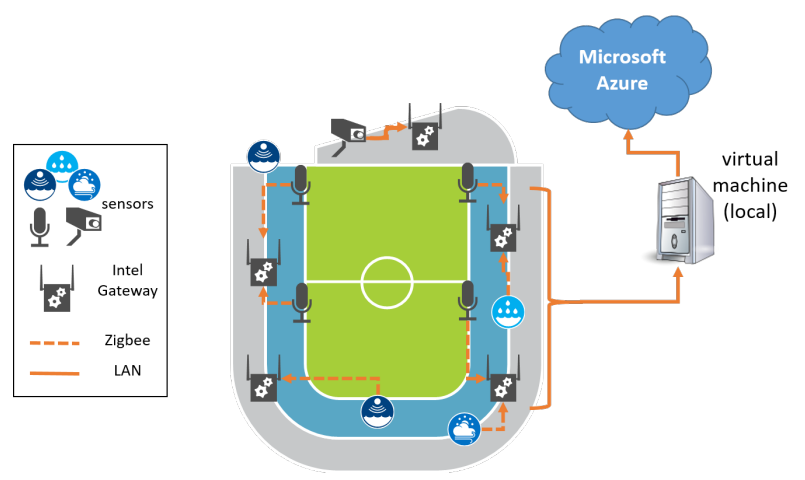

Figure 1: High-level architecture of the current testbed

when the stadium was near capacity. However a highly successful end-to-end test was conducted that resulted in sound level data being presented on the big screen at half-time and engaging with fans via social media.

We briefly outline related work in smart testbeds in section 2 . The general use cases that have been explored in the Smart Stadium project are described in section 3.1 followed by the current testbed architecture. The sound monitoring experiment - How loud is the $16^{\text {th }}$ player? - is described in section 4 and the data gathered is analysed in section 5 . The outcomes and learnings from this process and a brief description of the future plans conclude the paper.

\section{RELATED WORK}

Many stadiums are labelled as "smart" but for a successful IoT testbed more is required than high quality, full coverage Internet connectivity. Stadiums such as the Amsterdam Arena with its innovation lab (http://amsterdaminnovationarena.com/) and Levi's Stadium in the US [2] are both stadiums that are pushing innovations in smart technologies focussed on commercial and near-to-market outcomes beyond connectivity. These stadiums strive to develop solutions for improved fan engagement, better retail solutions and efficient environmental performance.

Other IoT testbeds have been proposed or developed in the context of cities [12], campuses [9], marine environments [11] and urban climate monitoring [1]. These provide excellent opportunities to develop and evaluate solutions for specific problems and are complementary to the goals of the Smart Stadium project. Within the EU, the Future Internet Testing facility IoT lab ${ }^{2}$ offers options to test wireless sensor devices and communication protocols at very large scale. There is also growing interest in linking silo'd test installations across smart cities (e.g., the Pervasive Nation project in Ireland, http://pervasivenation.ie) to encourage interoperability of systems and data semantics [5].

The beauty of a stadium testbed is that Croke Park is much more than just a sporting venue. It hosts events attended by $82,000+$ people including sport, concerts, graduations and other public events. It is a conference venue, a tourist destination, a retail and business hub. As the use cases described in the next section illustrate, there

\footnotetext{
${ }^{2}$ https://www.iot-lab.info/
}

are many opportunities to trial technologies at multiple levels of commercial readiness in a controlled, yet realistic, environment.

The major challenges identified in IoT research focus on the communication technology (networks, protocols, hardware, etc.), the data technology (standards, formats, storage) and the semantic fusion (analytics, creating meaning, etc.) $[3,13,14]$. There are numerous proposed platforms and technologies that improve the way in which IoT systems can be constructed and deployed [8]. Creating the testbed facility within the Smart Stadium project required using the technology, choosing the installation locations, developing the scripts to capture and upload data packets and learning about the practical challenges of conducting an end-to-end process within the testbed.

A very recent and thorough survey on the requirements and challenges for smart cities [3] highlights many of the same circumstances that can be evaluated and explored in a stadium, acting as a microcosm of a smart city. Particularly the opportunities to explore questions around robustness, security, personal privacy and data analytics. The end-to-end tests conducted around the sound level monitoring within Croke Park have enabled us to explore and learn lessons in installing, configuring and utilising internet-of-things technologies in a real-world situation.

\section{STADIUM TESTBED}

\subsection{Use cases}

\section{Sound Monitoring}

Croke Park Stadium is positioned within an urban area. To capture noise levels throughout the stadium, sound monitoring equipment was positioned at four points. Two within the stadium at the stands and two outside the stadium. This allowed us to measure crowd cheer within the stadium but also compare this to external sound to monitor noise pollution for neighbouring areas. The photo in Figure 2 shows the position of one of the Sonitus microphones commonly used to measure sound at industrial and construction sites. Sound data is measured by the microphones and averaged over a 1 minute period and then sent to the closest gateway where it is then sent to the cloud via a master gateway.

An automated monitoring solution enables reduced overhead. The existing solution is very manual and requires significant effort throughout a concert to record results. Automated microphones are always running meaning that all events are captured and enabling a solid historical baseline for comparison. Additionally sound data can be disseminated through multiple channels, e.g. a website, a publicly accessible app or a dashboard for key personnel.

Weather Monitoring

Croke Park's Skyline tour offers spectacular views of the city from on top of the stadium where it can be very windy. By monitoring wind speeds, stadium staff can make informed decisions on whether conditions are optimal for patrons. A weather station on the top of the stadium roof is used to monitor a number of meteorological metrics including wind speed.

Pitch Monitoring

The pitch at Croke Park is widely regarded as one of the best in the world. It's also one of the busiest with nearly 90 events per year. A lot of effort is required to maintain the pitch because it's twice the size of a conventional soccer field. Part of the pitch is in the shadow 


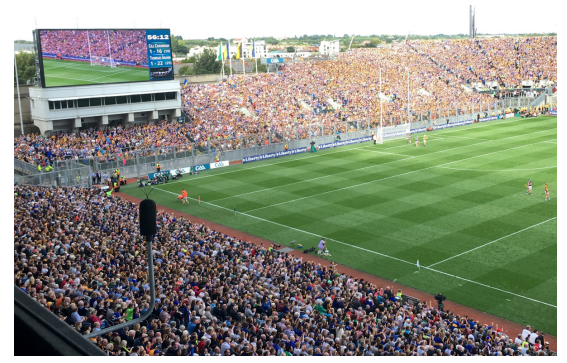

Figure 2: Microphone in place in stadium

created by the stadium much of the year - a common problem for stadium surfaces. To stimulate grass growth and ensure the best possible playing surface, the staff deploys heat lamps, which are very costly to run. Part of the Smart Stadium project involves monitoring the best environment for grass growth using a fixed camera to track sunlight exposure with the aim of optimising the use of the heat lamps.

Understanding the Crowd

Finally, an area of great interest is improving technologies to understand how a crowd moves through a designated area such as a stadium or street scape. Using existing CCTV cameras, we have developed algorithms to detect and estimate the density of a crowd and to annotate crowd activities [6, 7]. Within the stadium this is useful for improving mobility, security and logistics. For example, understanding how people move between their seats, the concession and rest areas at half-time. Until recently this has been a manual process of retrieving video footage and processing offline but the next steps will fully integrate the algorithms onto an upgraded gateway for video processing and analytics closer to the point of capture.

\subsection{Testbed Architecture}

Figure 1 shows the general layout of the equipment in the stadium and Table 1 lists the details of the current installation. The testbed was created to support a broad number of possible applications, some of which have been mentioned in the use cases section. The purpose of this project was primarily research and some decisions made were done so in this light. For example, choice of radio and gateway devices would be different when this project is rolled out for full production. This said, conducting this research project with a clear view of the business models and use cases from the outset has enabled all stakeholders to learn exactly what is involved in developing and deploying an IoT solution that can potentially drive business value and provide a productive research testbed.

The fundamental premise of the technical solution was to design with the following architectural concepts in mind.

Loosely coupled components

This meant that each component used was not dependant on any other component or could easily be replaced, updated or removed without affecting the entire solution. The benefit of this approach was that the team could test individual components and replace or update independently when required.

Queue centric approach

Following on from the loosely coupled approach the project team wanted to build as much resilience into the solution as possible. For

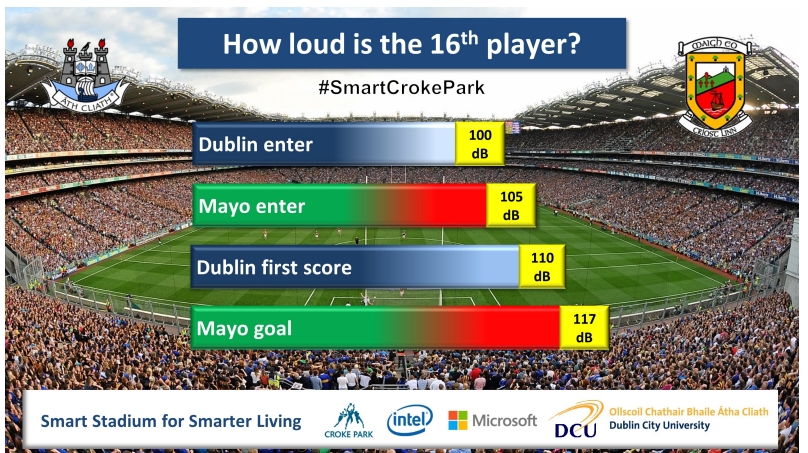

Figure 3: Half-time crowd sound analysis, 2016 football final replay, Dublin vs Mayo

example, one of the challenges within the project was positioning of microphones in relation to the radio antenna used to provide connectivity for the microphone gateways back to the master gateway which communicated with the cloud. Messages sent to master gateway are forwarded to the cloud using the IoT hub. If there is an issue with connectivity this data is still stored on Sonitus system and logged on the gateway. This same principle was adopted in the cloud where different services performed separate functions and communicated with each other via queues.

Separation of Concerns

Each component was designed to provide only a specific function. This again supports the maintainability and extensibility of the solution by allowing each component to be updated without affecting the entire solution. This proved critical in this IoT project as there are so many components doing different things. The best example of this is separating ingestion from real time communications via the IoT Hub and Stream Analytics in the Azure cloud. When we wanted to update or amend a new real time query to the data we could stop the stream analytics job without affecting the ingestion and update the queries before restarting the service. This is fundamental to creating a solution that could scale to a globally deployed IoT scenario.

\section{HOW LOUD IS THE $16^{\mathrm{TH}}$ PLAYER?}

Gaelic games ${ }^{5}$ (football, hurling, camogie) are played by teams of 15 players each. Informally the ability of supporters to cheer their team on to victory is sometimes referred to as the effect of the " $16^{\text {th }}$ player". The general sound use case described in section 3.1 presents a strong commercial case for employing sound level monitoring. However there is also a good fan engagement purpose. A prior collaboration with DCU, Intel, Arizona State University (ASU) and the Sun Devil Stadium located on ASU campus had explored the idea of a "Victory Cheer" where sections of the crowd competed via an instrumented stadium and supporter's app to win prizes based on the volume of their cheering [10].

The Croke Park testbed is designed to support the full process from sensor reading to gateway to virtual machine to cloud analytics to public communication (Figure reffig:arch). To test this the experimental system developed to allow the park to measure the

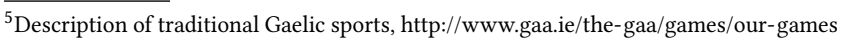


Table 1: Smart Stadium Testbed Equipment (Phase I, 2016)

\begin{tabular}{|c|c|c|c|}
\hline Component & Provider & Details/Links & Notes \\
\hline Sound monitoring equipment & Sonitus Systems & EM2010 sound level monitor ${ }^{3}$ & $\begin{array}{l}4 \text { sound monitoring microphones positioned around the } \\
\text { stadium, } 2 \text { internal and } 2 \text { external as per stadium map } \\
\text { (Figure 1). }\end{array}$ \\
\hline Connectivity & Intel/Sonitus & Seedstudio RFBee v1.1 & $\begin{array}{l}\text { Operating at } 868 \mathrm{MHz} \text {. Each unit is set up in Transceive } \\
\text { mode (Send and receive), Baud rate } 96008 \mathrm{~N} 1 \text {, no flow } \\
\text { control, attached via USB connection and using UartS- } \\
\text { Bee adapter. Data is transferred in wireless serial mode } \\
\text { using UART between the RFBee unit and the Gateway. }\end{array}$ \\
\hline Gateway Devices & Intel & SuperMicro E100-8Q & $\begin{array}{l}5 \text { Intel Quark }{ }^{\mathrm{TM}} \text { gateways positioned strategically } \\
\text { around the stadium based on the networking access } \\
\text { and line of sight between sensors and gateways within } \\
\text { optimal transmission distances for the RFBee radios. }\end{array}$ \\
\hline Master Gateway & $\mathrm{DCU}$ & Ubuntu 16.04 VM & $\begin{array}{l}\text { This machine aggregates and collates all data from the } \\
\text { gateways to be pushed securely to the cloud. }\end{array}$ \\
\hline
\end{tabular}

${ }^{2}$ http://www.sonitussystems.com/products/EM2010 ${ }^{3}$ https://azure.microsoft.com/en-us/develop/iot/

averaged noise levels outside the stadium for regulatory compliance was re-purposed to create a friendly fan competition within the stadium bowl. Microphones capture maximum decibel peaks in crowd cheering levels and gateways send this information to the Azure IoT hub. Data is presented on a dashboard to staff, who in turn project it on the stadium screens enabling them to "gamify" the data and identify which section is making the most noise. The data was presented during the 2016 All-Ireland Hurling and Football Finals and compared the noise levels at particular points in the games focussing on the entry of each team and key scoring moments as a proxy for measuring team supporter's excitement ${ }^{6}$. Figure 3 was created and displayed on the stadium screens at half-time.

As can be seen in Figure 3, four peak readings were selected that correlated with specific events during the match - the entrance of each team onto the pitch, the first score by Dublin and a goal by the Mayo team. The noise resulting from the Mayo goal (worth more points than a score) was the loudest reading observed at the stadium during the football season $(117 \mathrm{~dB}$, roughly equivalent to an emergency vehicle siren or a clap of thunder).

To produce the crowd sound analysis, firstly the two internal microphones register the decibels levels and create per minute summaries of the maximum and average $\mathrm{dB}$ which would be packaged and transmitted via Zigbee to the nearest gateway device. The gateway would process the incoming packets into JSON format (see sample in Figure 4) and transmit that via a virtual network on the stadium LAN to the virtual machine running the master gateway to initiate the secure transfer to the Azure cloud platform. The services on Azure would store and analyse the incoming data streams to create a dashboard showing both the per minute and a 15 minute rolling average of the sound levels. A controller on

${ }^{6}$ Local coverage of the sound measurement, http://www.thejournal.ie/ croke-park-noise-levels-2987003-Sep2016/

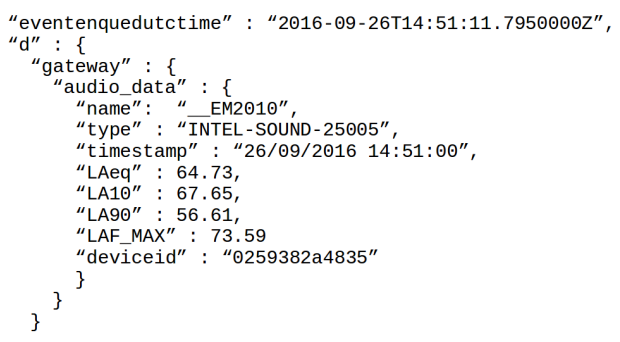

Figure 4: Example JSON packet for transmission to cloud

the day, watching the match, would access this data and build the graphic to display on the screen at half time.

Performing sound analysis in this way has a number of major limitations. Allocating sound level readings to particular events is somewhat inexact With only two data points and no segregation of fans within Croke Park stadium. The overall outcomes of this exercise - to test the systems and engage with the public - are unaffected by these constraints but they are worth noting. Firstly, there is only indirect evidence linking the observed events on the pitch to the maximum sound volume reached. For example the loudest point occurred during the Mayo goal but is equally composed of groans from the Dublin supporters as cheers from the Mayo supporters.

Secondly the granularity of the sound data packets (1 measurement per minute) is insufficient for events that occur during a sporting event. A single minute can easily contain multiple scoring attempts or other contentious actions on the pitch and it's not possible to allocate a maximum reading precisely. For example, in other matches the teams entered the pitch within 60 seconds of each and it wasn't possible to assign a "crowd sound" level to each team's supporters. The 1 minute windows were originally selected 


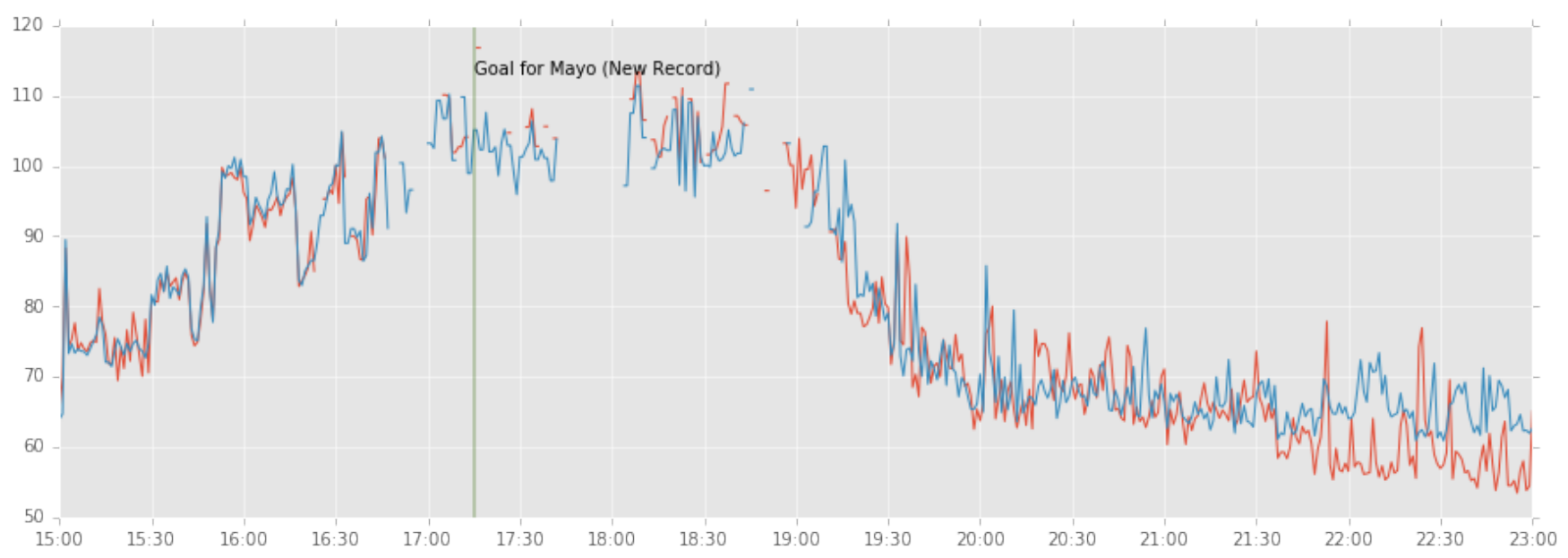

Figure 5: Maximum sound levels (in $\mathrm{dB}$ ) taken from internal microphones during the match

to supply more detailed data than the standard configuration of 15 minutes windows that are used for regulatory compliance in measuring construction sites. The 1 minute readings are used to compile a sliding 15 minute window on the Azure dashboard.

Thirdly the positioning of the microphones may indirectly influence the observations due to the position of corporate boxes immediately above the microphone. It's possible that this section of the crowd is quieter or less partisan. The positions of the microphones were chosen originally to maximise bowl coverage from the available number of microphones and to make best use of the available gateway devices. Some installation decisions were also due to the easy availability of existing power and network connectivity within the stadium.

Finally, differences in maximum sound levels are often very small (1-2 dB) which makes gamification of the sound measurements quite difficult. Increasing microphone coverage of the stadium and altering the granularity of the readings would help to overcome some of these limitations.

\section{MATCH DAY PACKET LOSS ANALYSIS}

Figure 5 shows the maximum decibels levels observed for a period starting just prior to the beginning of the 2016 football final. The shape of a match is clearly defined, with a gradual increase as the crowd files in from around 3pm, throw in (GAA equivalent to kickoff) at 4:30pm and the sharp decrease in sound levels as the match ends and the crowd rapidly leaves the area. There are also a number of gaps where significant packet loss occurred and data didn't reach the Azure data store.

Loss of data packets from the sound level monitoring system was experienced especially during big events when the stadium is filled with over 82,000 fans. Figures 6 and 7 visualise the loss of packets on game day from the two internal microphones and show the clear loss that occurs during a match and especially at half-time. We speculate that the sharp loss of data that occurs during halftime is due to the abrupt increase in patrons using wifi and mobile data connections and the increased movement of people creating barriers of warm bodies that interfere with packet transmission

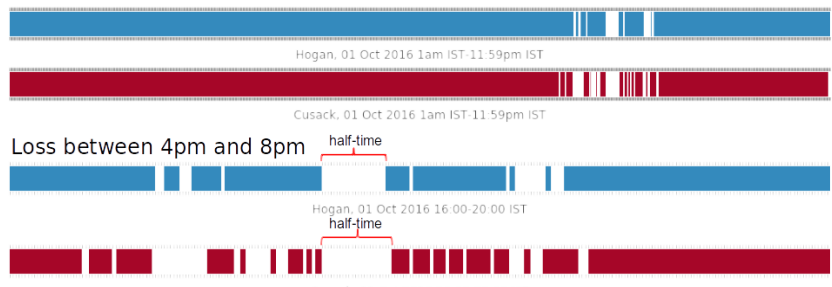

Figure 6: Packet loss $1^{\text {st }}$ Oct 2016

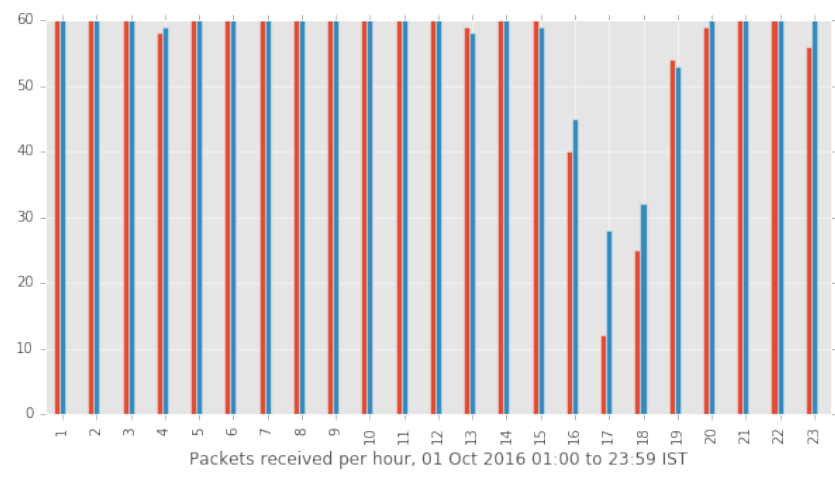

Figure 7: Packet loss during the football match

over Zigbee. This is an issue that is not unique to this deployment. Mobile carriers and internet providers have spent decades to find a solution to establish fast and reliable wireless data communication channels in this kind of highly dense and dynamic environment.

It was difficult initially to understand where the loss occurred, however, after investigation, two main issues were uncovered:

Signal interference The current setup may suffer from radio signal interference in the high dynamic and high density environment. The motion from human bodies and the signal from their mobile devices along with wireless signals from TV broadcast crews, Garda 
(Irish police) officers, security teams may all interfere the wireless data communication between the microphone and the gateway. This can be confirmed (from the graphs) that the number of packets lost starts decreasing when the game is over and almost no packets were lost as soon as all the fans exited the stadium. This issue is well recognised and a project is underway to build more resilience into to the system.

ISON format The Azure backend setup relies on data being sent in valid JSON format before being analysed and aggregated in real time. Any invalid value or changes to the format resulted in dropped packets by Azure. New functionality in Stream Analytics makes it very easy to test real time analytics scripts against sample data, but there seems to be no real way of dealing with invalid formats. To resolve this all data was pushed to BLOB storage so that we could analyse all packets to understand where formats were changing. This helped greatly in agreeing a strict format and sticking with it as well as making the stream analytics jobs flexible enough to handle new data sent within the packets. Invalid JSON was much more difficult to deal with and is simply dropped at the moment. A current update to the gateways will validate JSON formats before sending to Azure and alerting or logging results. In addition, a two-way communication mechanism may be applied so that Azure can request the gateway to re-send the missing/damaged data at quieter times.

\section{CONCLUSIONS AND FUTURE WORK}

The Smart Stadium testbed at Croke Park provides a system that is small enough to develop and trial new IoT technologies but large enough to prove and evaluate systems. The end-to-end test that was performed as part of the "How loud is the $16^{\text {th }}$ player?" activity enabled us to learn about the strengths and weaknesses of the gateways, Zigbee and network systems. We discovered that significant packet loss from sensor to cloud occurs due to both hardware and software restrictions and are currently installing new systems with stronger antennae and better line of sight positioning to test varying connectivity settings. We re-purposed commercial sound level monitoring systems to run a public engagement activity for better understanding of IoT and smart systems technologies.

There is significant current and future work ongoing within the Smart Stadium for Smarter Living project. The use cases described in section 3.1 are continuing to create data that is being analysed. Phase II equipment is currently being rolled out and the lessons learned about installation locations, network and power requirements and data security are being applied to improve the testbed's flexibility and resilience. We look forward to continuing to develop and apply practical prototypes to overcome research gaps in IoT systems.

\section{ACKNOWLEDGMENTS}

The authors would like to acknowledge the support of Croke Park in hosting the testbed. The work is funded in part by a Microsoft Research Grant to use the Azure platform and in part from Science Foundation Ireland (SFI) under Grant Number SFI/12/RC/2289 (Insight Centre for Data Analytics).

\section{REFERENCES}

[1] Lee Chapman, Catherine L. Muller, Duick T. Young, Elliott L. Warren, C. S. B. Grimmond, Xiao-Ming Cai, and Emma J. S. Ferranti. 2015. The Birmingham Urban Climate Laboratory: An Open Meteorological Test Bed and Challenges of the Smart City. Bulletin of the American Meteorological Society 96, 9 (2015), $1545-1560$.

[2] Intel Corporation. 2016. Smart Stadiums Take the Lead in Profitability, Fan Experience, and Security. Technical Report. https://www.intel.com/content/www/us/ en/internet-of-things/solution-briefs/iot-smart-stadiums-brief.html

[3] Ammar Gharaibeh, Mohammad A. Salahuddin, Sayed J. Hussini, Abdallah Khreishah, Issa Khalil, Mohsen Guizani, and Ala Al-Fuqaha. 2017. Smart Cities: A Survey on Data Management, Security and Enabling Technologies. IEEE Communications Surveys \& Tutorials (2017), 1-1. https://doi.org/10.1109/COMST.2017. 2736886

[4] Alexander Gluhak, Srdjan Krco, Michele Nati, Dennis Pfisterer, Nathalie Mitton, and Tahiry Razafindralambo. 2011. A survey on facilities for experimental internet of things research. IEEE Communications Magazine 49, 11 (2011), 58-67. https: //doi.org/10.1109/MCOM.2011.6069710

[5] Jorge Lanza, Luis Sanchez, David Gomez, Tarek Elsaleh, Ronald Steinke, and Flavio Cirillo. 2016. A Proof-of-Concept for Semantically Interoperable Federation of IoT Experimentation Facilities. Sensors 16, 7 (June 2016), 1006. https://doi.org/ 10.3390/s16071006

[6] Mark Marsden, Kevin McGuinness, Suzanne Little, and Noel E O'Connor. 2016. Holistic features for real-time crowd behaviour anomaly detection. In Image Processing (ICIP), 2016 IEEE International Conference on. 918-922.

[7] Mark Marsden, Kevin McGuinness, Suzanne Little, and Noel E O'Connor. 2017. ResnetCrowd: A Residual Deep Learning Architecture for Crowd Counting, Violent Behaviour Detection and Crowd Density Level Classification. In 14th IEEE International Conference on Advance Video and Signal-based Surveillance (AVSS).

[8] Julien Mineraud, Oleksiy Mazhelis, Xiang Su, and Sasu Tarkoma. 2016. A gap analysis of Internet-of-Things platforms. Computer Communications 89 (2016), 5-16. https://doi.org/10.1016/j.comcom.2016.03.015

[9] Michele Nati, Alexander Gluhak, Hamidreza Abangar, and William Headley. 2013. SmartCampus: A user-centric testbed for Internet of Things experimentation. In Wireless Personal Multimedia Communications (WPMC), 2013 16th International Symposium on. 1-6.

[10] Sethuraman Panchanathan, Shayok Chakraborty, Troy McDaniel, Ramin Tadayon, Bijan Fakhri, Noel E. O'Connor, Mark Marsden, Suzanne Little, Kevin McGuinness, and David Monaghan. 2017. Enriching the Fan Experience in a Smart Stadium Using Internet of Things Technologies. International fournal of Semantic Computing 11, 2 (June 2017), 137-170.

[11] Fiona Regan, Timothy Sullivan, Ciprian Briciu, Helen Cooney, Kevin Murphy, Dian Zhang, Edel O Connor, Noel O Connor, and Alan Smeaton. 2013. A Smart City-Smart Bay Project - Establishing an Integrated Water Monitoring System for Decision Support in Dublin Bay. (2013), 75-82.

[12] Luis Sánchez, Verónica Gutiérrez, José Antonio Galach, Pablo Sotres, Juan Ramón Santana, Javier Casanueva, and Luis Muñoz. 2013. SmartSantander: Experimentation and Service Provision in the Smart City. 16th International Symposium on Wireless Personal Multimedia Communications (2013), 4-9.

[13] Dhananjay Singh, Gaurav Tripathi, and Antonio J. Jara. 2014. A survey of Internet-of-Things: Future vision, architecture, challenges and services. 2014 IEEE World Forum on Internet of Things, WF-IoT 2014 (2014), 287-292. https: //doi.org/10.1109/WF-IoT.2014.6803174

[14] Andrew Whitmore, Anurag Agarwal, and Li Da Xu. 2015. The Internet of Things A survey of topics and trends. Information Systems Frontiers 17, 2 (2015), 261-274. https://doi.org/10.1007/s10796-014-9489-2 